\title{
EDITORIAL
}

\section{NEW MIGRAINE PROPHYLACTIC DRUG OPTIONS}

\author{
José Luiz Dias Gherpelli
}

RHCFAP/3101

GHERPELLI JLD - New migraine prophylactic drug options. Rev. Hosp. Clín. Fac. Med. S. Paulo 57(6):249-250, 2002.

The review article published in this issue, concerning new options for preventive drug treatment of migraine, deals with a subject that always draws the attention of both general practitioners and neurologists because of the high frequency with which they are confronted with doubts related to treatment in everyday practice. Several drugs are reported to be useful and consequently are used for both acute and preventive treatment of migraine attacks, with a wide range of efficacy between them that probably arises from different individual responses, which arise from different pathophysiologic factors that may be involved in each case. Several drugs have been tested for their therapeutic efficacy in wellconducted, double-blind, placebo-controlled clinical trials. Nevertheless, patients often have a disappointing clinical response. From time to time, review articles are published on the subject ${ }^{1,2}$, and although they suggest that several drugs are efficient in decreasing migraine frequency and/or intensity, they also show that we are far from identifying the ideal drug. This failure is most certainly related to our poor understanding of the basic disease mechanisms. Perhaps it would be more appropriate to address migraine as a group of closely related entities, since there are different types of migraine (with differences in the intensity, triggering, and associated factors, presence or absence of the different types of aura, etc.), probably with different pathophysiologic etiologies.

Even the mechanisms of action of the various drugs that are used in preventive migraine treatment are not fully understood.

The placebo effect is particularly high, not only in preventive therapy, but also in the acute phase, ranging from $20 \%$ to $70 \%$, according to reports in the literature ${ }^{4}$. This fact only adds to the difficulties in studying drug efficacy in a disease in which psychosomatic or other environmental factors seem to play such an important role in its symptomatic expression. Therefore, even studies that adhere to the strict rules of scientific research must be interpreted with caution when they test a new drug option. Other investigators must retest these drugs before they can be accepted as really useful. The review article reports on the efficacy of

From the Child and Adolescent Headache Clinic of the Department of Neurology, Hospital das Clínicas, Faculty of Medicine, University of São Paulo. several drugs that have been tested and still have not been widely used in preventive migraine therapy.

Reports on the efficacy of riboflavin and magnesium salts were originally published some years ago. These are low cost-therapies and ideally should be studied further to make sure that their efficacy is reliable, and their adverse-effect rate is low enough to allow them to be incorporated into our prescriptions.

The efficacy of antiepileptic drugs in preventive migraine therapy is a long story that waxes and wanes over the time. Several of the traditional drugs, such as phenobarbital, phenytoin, carbamazepine, and more recently, divalproate, were or are still used, with efficacy reported eventually for all of them over the years, but seldom proven, except regarding divalproate. The new antiepileptic drugs-gabapentin, topiramate, zonisamide, tiagabine, and levetiracetam, which were reviewed in the article in this issue-have only scant data on their efficacy (most of the references cited were published as abstracts). Lamotrigine, another recently marketed antiepileptic drug, has also been tested, and it proved ineffective with migraines ${ }^{3}$. On the other hand, all 
these newer drugs are much more expensive than the traditional drugs used nowadays, a problem that is particularly important in developing countries. The high-cost problem also will limit the use of botulinum toxin.

Concerning the other drugs, montelukast and lisinopril, their efficacy and place in migraine treatment is welcome if proven over the years, because positive results following treatment with these drugs will also add to our understanding of the basic pathophysiologic mechanisms of the disease.

\section{REFERENCES}

1. ADELMAN JU, BROD A, VON SEGGERN RL et al. - Migraine preventive medications: a reappraisal. Cephalalgia 1998, 18: 605-11.

2. SILBERSTEIN SD - Preventive treatment of migraine: an overview. Cephalalgia 1997, 17: 67-72.
3. STEINER TJ, FINDLEY LJ \& YUEN AW - Lamotrigine vs. placebo in the prophylaxis of migraine with and without aura. Cephalalgia 1997, 17: 109-12.

4. TFELT-HANSEN P, BOUSSER M-G, SOLOMON S et al. Guidelines for controlled trials of drugs in migraine. In: The IHS Member's Handbook 2000. Stockholm: Scandinavian Univ Press, 1999, p. 111-33. 